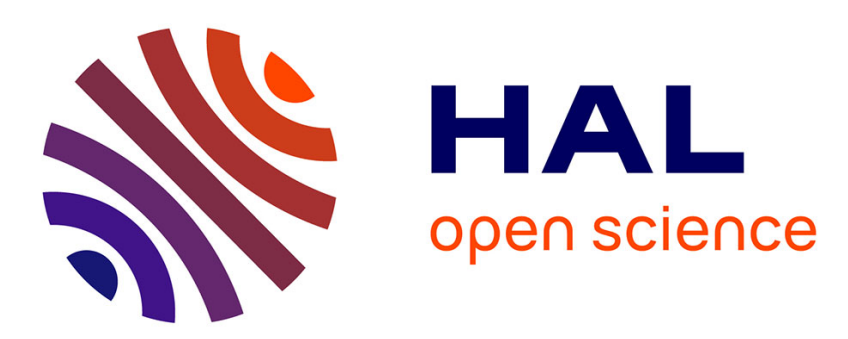

\title{
A Numerical Approach to the Optimal Control and Efficiency of the Copepod Swimmer
}

Bernard Bonnard, Monique Chyba, Jérémy Rouot, Daisuke Takagi

\section{To cite this version:}

Bernard Bonnard, Monique Chyba, Jérémy Rouot, Daisuke Takagi. A Numerical Approach to the Optimal Control and Efficiency of the Copepod Swimmer. 55th IEEE Conference on Decision and Control - CDC, Dec 2016, Las Vegas, United States. pp.4196-4201, 10.1109/CDC.2016.7798906 . hal-01286602v3

\section{HAL Id: hal-01286602 https://hal.inria.fr/hal-01286602v3}

Submitted on 24 Oct 2016

HAL is a multi-disciplinary open access archive for the deposit and dissemination of scientific research documents, whether they are published or not. The documents may come from teaching and research institutions in France or abroad, or from public or private research centers.
L'archive ouverte pluridisciplinaire HAL, est destinée au dépôt et à la diffusion de documents scientifiques de niveau recherche, publiés ou non, émanant des établissements d'enseignement et de recherche français ou étrangers, des laboratoires publics ou privés. 


\title{
A Numerical Approach to the Optimal Control and Efficiency of the Copepod Swimmer
}

\author{
B.Bonnard and M.Chyba and J.Rouot and D.Takagi
}

\begin{abstract}
This article presents a geometric and numerical approach to compute the optimal swimming strokes of a larval copepod. A simplified model of locomotion at low Reynolds number is analyzed in the framework of Sub-Riemannian geometry. Both normal and abnormal geodesics are considered along which the mechanical power dissipated by the swimmer is conserved. Numerical simulations show that, among various periodic strokes, a normal stroke consisting of a simple loop shape is maximizing the efficiency.
\end{abstract}

\section{INTRODUCTION}

Swimming microorganisms employ a variety of mechanisms of propulsion, and they have inspired numerous models starting with undulating sheets and filaments introduced in the fifties [11], [19]. Recent studies have explored optimal strategies for swimming with minimal amount of mechanical work, an important criterion for assessing the fitness of different organisms and for designing efficient robotic swimmers [13]. Previous studies have computed optimal solutions in the framework of variational analysis or optimal control [2], [4], [5], [18].

Recently a new model was developed to mimic the locomotion of larval copepods, an abundant type of zooplankton thriving in the ocean [15], [17]. The simplest form of the model, hereafter referred to as the copepod swimmer, is a symmetric body consisting of two pairs of legs, with the pairs making respectively an angle $\theta_{1}$ and $\theta_{2}$ with respect to the displacement direction $O x$ (see Fig.1).

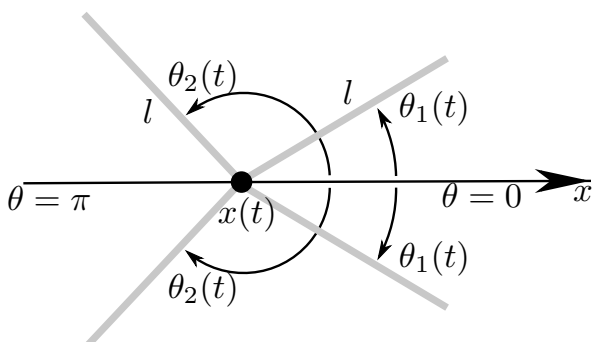

Fig. 1. Copepod swimmer

J. Rouot is supported by the French Space Agency CNES, R\&T action R-S13/BS-005-012 and by the région Provence-Alpes-Côte d'Azur

M. Chyba and D. Takagi: 2565 McCarthy the Mall, Department of Mathematics, University of Hawaii, Honolulu, HI 96822, USA chyba@hawaii.edu, dtakagi@hawaii.edu

Bernard Bonnard and Jeremy Rouot: Inria Sophia Antipolis and Institut de Mathématiques de Bourgogne, 9 avenue Savary, 21078 Dijon, France bernard.bonnardau-bourgogne.fr, jeremy . rouoteinria.fr
The swimming velocity is given by [17]

$$
\dot{x}=\frac{\dot{\theta}_{1} \sin \theta_{1}+\dot{\theta}_{2} \sin \theta_{2}}{2+\sin ^{2} \theta_{1}+\sin ^{2} \theta_{2}}
$$

and the controls are the angular velocities

$$
\dot{\theta}_{1}=u_{1}, \quad \dot{\theta}_{2}=u_{2} \text {. }
$$

We also have the state constraints $\theta_{i} \in[0, \pi], i=1,2$, and $\theta_{1} \leq \theta_{2}$.

The true cost corresponding to the mechanical energy of the system is given by the quadratic form $\dot{q}^{t} M \dot{q}$ where $q=$ $\left(x, \theta_{1}, \theta_{2}\right)$ and

$$
M=\left(\begin{array}{ccc}
2-\frac{1}{2}\left(\cos ^{2} \theta_{1}+\cos ^{2} \theta_{2}\right) & -\frac{1}{2} \sin \theta_{1} & -\frac{1}{2} \sin \theta_{2} \\
-\frac{1}{2} \sin \theta_{1} & \frac{1}{3} & 0 \\
-\frac{1}{2} \sin \theta_{2} & 0 & \frac{1}{3}
\end{array}\right)
$$

Using (1), the optimization problem is equivalent to minimizing the quadratic cost

$$
\int_{0}^{T} a(q) u_{1}^{2}+2 b(q) u_{1} u_{2}+c(q) u_{2}^{2} \mathrm{~d} t
$$

with

$$
\begin{aligned}
& a=\frac{1}{3}-\frac{\sin ^{2} \theta_{1}}{2\left(2+\sin ^{2} \theta_{1}+\sin ^{2} \theta_{2}\right)}, \\
& b=\quad-\frac{\sin \theta_{1} \sin \theta_{2}}{2\left(2+\sin ^{2} \theta_{1}+\sin ^{2} \theta_{2}\right)}, \\
& c=\frac{1}{3}-\frac{\sin ^{2} \theta_{2}}{2\left(2+\sin ^{2} \theta_{1}+\sin ^{2} \theta_{2}\right)} .
\end{aligned}
$$

This copepod swimmer serves as a suitable model for computing optimal controls in the framework of subRiemannian (SR) geometry. The system is three-dimensional (two shape variables and one displacement variable), which is arguably simpler than the five-dimensional system (two shape variables and three displacement variables) of the previously studied Purcell swimmer where the expression of the control fields is complicated (see [14]). It is a global model of SR-geometry which can be analyzed in detail, illustrating in particular the role of normal and abnormal geodesics in the motion. In addition, the optimal controls could be compared with observations of copepods to determine whether they are optimizing their strokes to minimize mechanical energy. Copepods must swim in order to find food and escape from predators, and they have had a chance to adapt and evolve over millions of years, but it remains unknown to what extent they have adapted their strokes to maximize their swimming efficiency. Thus the model optimization could offer new insight into biological behavior. 

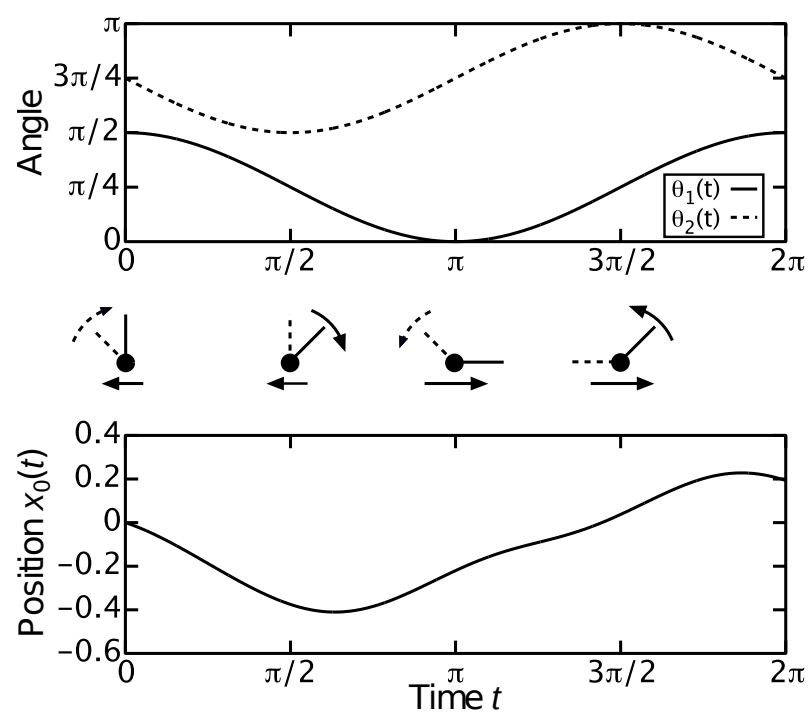

Fig. 2. Two legs oscillating sinusoidally according to $\theta_{1}=\pi / 4+a \cos t$ and $\theta_{2}=3 \pi / 4+a \cos (t+\pi / 2)$, where $a=\pi / 4$ is the amplitude. The second leg (blue) oscillates about $\Phi_{2}=3 \pi / 4$, while the first leg (red) oscillates about $\Phi_{1}=\pi / 4$ with a phase lag of $\pi / 2$. The swimmer position $x$ translates about a fifth of the leg length after one cycle.

This article is organized into two sections. Sections IIIII contain the contribution of this article. In section II, we recall some properties of the copepod swimmer [17] and the mathematical tools from geometric optimal control (see [7] for a general reference). This section is also devoted to geometric computations of the copepod swimmer to study the role of normal and abnormal strokes. In section III we present numerical simulations to describe the normal strokes in relation with the classification of periodic planar curves [3]. Finally, the optimal strokes satisfying the constraints are numerically computed using the two software: Bocop (www.bocop.org, [6]) and HamPath (http: // cots.perso.enseeiht.fr/hampath/, [9]).

\section{Preliminary Results}

\section{A. Periodic strokes}

A (general) stroke of period $T$ consists in a periodic motion in the shape variables $\left(\theta_{1}, \theta_{2}\right)$. Assuming $x(0)=0$, the corresponding displacement is $x(T)$. In [17], two types of geometric motions are described:

First case (Fig.2): The two legs are assumed to oscillate sinusoidally with period $2 \pi$ according to

$$
\theta_{1}=\Phi_{1}+a \cos (t), \quad \theta_{2}=\Phi_{2}+a \cos \left(t+k_{2}\right)
$$

with $a=\pi / 4, \Phi_{1}=\pi / 4, \Phi_{2}=3 \pi / 4$ and $k_{2}=\pi / 2$. This produces a displacement $x(2 \pi)=0.2$. Parameters $a, \Phi_{1}, \Phi_{2}$ and $k_{2}$ are designed to maximize the efficiency.

Second case (Fig.3): The two legs are paddling in sequence followed by a recovery stroke performed in unison. In this case the controls $u_{1}=\dot{\theta}_{1}, u_{2}=\dot{\theta}_{2}$ produce bang arcs to steer the angles from the boundary 0 of the domain to the boundary $\pi$, while the unison sequence corresponds to a displacement from $\pi$ to 0 with the constraint $\theta_{1}=\theta_{2}$.
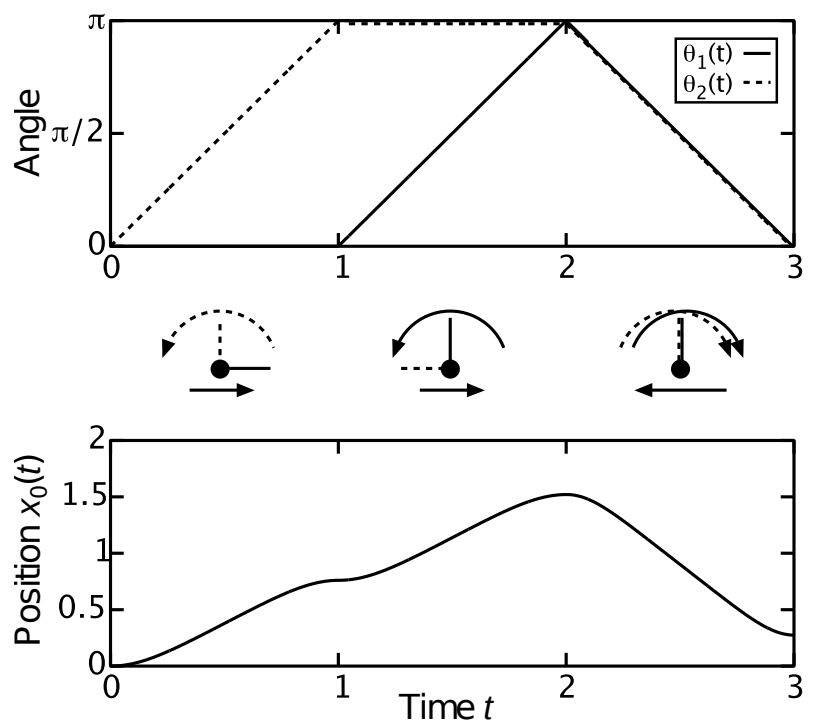

Fig. 3. Two legs paddling in sequence. The legs perform power strokes in sequence and then a recovery stroke in unison, each stroke sweeping an angle $\pi$.

Our main objective is to analyze these motions from the perspective of geometric optimal control.

\section{B. Abnormal curves}

With $q=\left(x, \theta_{1}, \theta_{2}\right)$, the system is written as a driftless affine control system

$$
\dot{q}(t)=\sum_{i=1}^{2} u_{i}(t) F_{i}(q(t))
$$

where the control vector fields are given by

$$
F_{i}=\frac{\sin \theta_{i}}{\Delta} \frac{\partial}{\partial x}+\frac{\partial}{\partial \theta_{i}}
$$

with $\Delta=2+\sin ^{2} \theta_{1}+\sin ^{2} \theta_{2}$. We denote by $D$ the distribution generated by the two vector fields: $D=\operatorname{span}\left\{F_{1}, F_{2}\right\}$.

The Lie bracket of two vector fields $F, G$ is computed with the convention

$$
[F, G](q)=\frac{\partial F}{\partial q}(q) G(q)-\frac{\partial G}{\partial q}(q) F(q) .
$$

Finally, we denote by $p=\left(p_{1}, p_{2}, p_{3}\right)$ the adjoint vector associated with $q$.

We first recall basic facts concerning the local classification of two-dimensional distributions in relation with abnormal curves. 
1) Local classification of two-dimensional distributions in dimension three and abnormal curves:

Let $D=\operatorname{span}\left\{G_{1}, G_{2}\right\}$ be the distribution generated by two vectors fields $G_{1}, G_{2}$ in $\mathbb{R}^{3}$. Let $z=(q, p)$ and denote $H_{i}(z)=\left\langle p, G_{i}(q)\right\rangle, i=1,2$ the Hamiltonian lifts. The Poisson bracket is given by

$$
\left\{H_{1}, H_{2}\right\}(z)=d H_{1}\left(\vec{H}_{2}\right)(z)=\left\langle p,\left[G_{1}, G_{2}\right](q)\right\rangle .
$$

Abnormal curves are defined by

$$
H_{1}(z)=H_{2}(z)=0
$$

and differentiating using the dynamics

$$
\frac{\mathrm{d} z}{d t}=\sum_{i=1}^{2} u_{i} \vec{H}_{i}(z)
$$

we obtain the relations

$$
\begin{aligned}
& \left\{H_{1}, H_{2}\right\}(z)=0 \\
& u_{1}\left\{\left\{H_{1}, H_{2}\right\}, H_{1}\right\}(z)+u_{2}\left\{\left\{H_{1}, H_{2}\right\}, H_{2}\right\}(z)=0
\end{aligned}
$$

defining the corresponding abnormal controls.

Tools from singularity theory can be used to classify the distributions, see [20]. Here we present only the two (stable) models related to our study.

Contact case. We say that $q_{0}$ is a contact point if $\left\{G_{1}, G_{2},\left[G_{1}, G_{2}\right]\right\}$ is of dimension three at $q_{0}$. At a contact point, identified to 0 , there exists a system of local coordinates $q=(x, y, z)$ such that

$$
D=\operatorname{ker}(\alpha), \quad \alpha=y \mathrm{~d} x+\mathrm{d} z .
$$

Observe that $\mathrm{d} \alpha=\mathrm{d} y \wedge \mathrm{d} x$ (Darboux form) and that $\frac{\partial}{\partial z}$ is the characteristic direction of $\mathrm{d} \alpha$. This form is equivalent to

$$
D=\operatorname{ker}\left(\alpha^{\prime}\right), \quad \alpha^{\prime}=\mathrm{d} z+(x \mathrm{~d} y-y \mathrm{~d} x),
$$

with

$$
\begin{aligned}
D & =\operatorname{span}\left\{G_{1}, G_{2}\right\}, & G_{1} & =\frac{\partial}{\partial x}+y \frac{\partial}{\partial z} \\
G_{2} & =\frac{\partial}{\partial y}-x \frac{\partial}{\partial z}, & G_{3} & =\left[G_{1}, G_{2}\right]=2 \frac{\partial}{\partial z} .
\end{aligned}
$$

The Martinet case. A point $q_{0}$ is a Martinet point if at $q_{0}$, $\left[G_{1}, G_{2}\right] \in D=\operatorname{span}\left\{G_{1}, G_{2}\right\}$ and at least one Lie bracket $\left[\left[G_{1}, G_{2}\right], G_{1}\right]$ or $\left[\left[G_{1}, G_{2}\right], G_{2}\right]$ does not belong to $D$. Then, there exist local coordinates $q=(x, y, z)$ near $q_{0}$ identified to 0 such that

$$
D=\operatorname{ker} \omega, \quad \omega=\mathrm{d} z-\frac{y^{2}}{2} \mathrm{~d} x,
$$

where

$$
\begin{aligned}
& G_{1}=\frac{\partial}{\partial x}+\frac{y^{2}}{2} \frac{\partial}{\partial z}, \quad G_{2}=\frac{\partial}{\partial y}, \quad G_{3}=\left[G_{1}, G_{2}\right]=y \frac{\partial}{\partial z} \\
& {\left[\left[G_{1}, G_{2}\right], G_{1}\right]=0, \quad\left[\left[G_{1}, G_{2}\right], G_{2}\right]=\frac{\partial}{\partial z} .}
\end{aligned}
$$

The surface $\Sigma: y=0$ where $G_{1}, G_{2},\left[G_{1}, G_{2}\right]$ are coplanar is called the Martinet surface and is foliated by abnormal curves, solutions of $\frac{\partial}{\partial x}$. In particular, through the origin it corresponds to the curve $t \rightarrow(t, 0,0)$.
2) Computations in the copepod case:

We have

$$
F_{3}=\left[F_{1}, F_{2}\right]=f\left(\theta_{1}, \theta_{2}\right) \frac{\partial}{\partial x}
$$

with

$$
\begin{aligned}
f\left(\theta_{1}, \theta_{2}\right) & =\frac{2 \sin \theta_{1} \sin \theta_{2}\left(\cos \theta_{1}-\cos \theta_{2}\right)}{\Delta^{2}}, \\
{\left[\left[F_{1}, F_{2}\right], F_{1}\right] } & =\frac{\partial f}{\partial \theta_{1}}\left(\theta_{1}, \theta_{2}\right) \frac{\partial}{\partial x} \\
{\left[\left[F_{1}, F_{2}\right], F_{2}\right] } & =\frac{\partial f}{\partial \theta_{2}}\left(\theta_{1}, \theta_{2}\right) \frac{\partial}{\partial x} .
\end{aligned}
$$

We deduce the following lemma.

Lemma 1: The singular set

$\Sigma:\left\{q ; \operatorname{det}\left(F_{1}(q), F_{2}(q),\left[F_{1}, F_{2}\right](q)\right)=0\right\}$, where the vector fields $F_{1}, F_{2},\left[F_{1}, F_{2}\right]$ are coplanar, is given by $2 \sin \theta_{1} \sin \theta_{2}\left(\cos \theta_{1}-\cos \theta_{2}\right)=0$ which corresponds to

- $\theta_{1}=0$ or $\pi$,

- $\theta_{2}=0$ or $\pi$,

- $\theta_{1}=\theta_{2}$.

It is formed by the boundary of the physical domain: $\theta_{i} \in[0, \pi], \theta_{1} \leq \theta_{2}$, with respective controls $u_{1}=0, u_{2}=0$ or $u_{1}=u_{2}$.

Remark 1: The previous lemma provides the interpretation of the policy represented in Fig.3. In the shape space $\left(\theta_{1}, \theta_{2}\right)$ it corresponds to a triangle. The edges of the triangle are abnormal curves (where by definition the linearized system is not controllable).

Remark 2: A recent contribution [12] proves that a trajectory with a corner of this type cannot be optimal (not taking into account the state constraints).

To analyze the first situation of Fig.2, the mechanical energy has to be used in relation with SR-geometry.

\section{Sub-Riemannian geometry}

The problem is written

$$
\dot{q}=\sum_{i=1}^{2} u_{i} G_{i}(q), \quad \min _{u(.)} \int_{0}^{T}\left(u_{1}^{2}+u_{2}^{2}\right) \mathrm{d} t,
$$

where the cost is defined for a fixed final time $T$. In this representation, we assume that the vector fields $G_{1}, G_{2}$ are orthonormal.

The Pontryagin Maximum Principle. The admissible controls are bounded measurable mappings. According to the Pontryagin maximum principle, we introduce the pseudoHamiltonian in the normal case

$$
H(z, u)=\sum_{i=1}^{2} u_{i} H_{i}(z)-\frac{1}{2} \sum_{i=1}^{2} u_{i}^{2},
$$

where the $H_{i}$ 's are the Hamiltonian lifts $\left\langle p, G_{i}(q)\right\rangle$. The maximization condition is equivalent to $\frac{\partial H}{\partial u_{i}}=0, i=1,2$. It follows that $u_{i}=H_{i}$ and plugging this expression for $u_{i}$ into $H$ produces the true Hamiltonian in the normal case

$$
H_{n}=\frac{1}{2}\left(H_{1}^{2}+H_{2}^{2}\right) \text {. }
$$


Definition 2: A normal stroke is a solution of $\overrightarrow{H_{n}}$ such that $\theta_{1}$ and $\theta_{2}$ are periodic with period $T$.

According to the transversality conditions of the maximum principle the adjoint variables $p_{2}$ and $p_{3}$ are such that $p_{2}$ and $p_{3}$ are both periodic with period $T$ (to produce a smooth solution).

Second order optimality condition. In the normal case, the first conjugate point corresponds to the first point where a normal geodesic ceases to be optimal with respect to the $C^{1}$ topology on the set of curves with fixed endpoints conditions. They can be computed using the HamPath software [9].

This leads to the following definition.

Definition 3: A normal stroke is called strict if it is not the projection of an abnormal extremal.

Lemma 4: A necessary optimality condition for a strict normal stroke to provide a weak minimizer is the nonexistence of a conjugate point on $] 0, T[$.

\section{Computations and analysis for the copepod swimmer}

We start by considering the simplified cost

$$
\min _{u(.)} \int_{0}^{T}\left(u_{1}^{2}+u_{2}^{2}\right) d t
$$

in relation with the contact case. Some geometric computations can be made, linked to the Heisenberg case. They can be used in the numerical implementation, in particular to compute strokes with small amplitudes. In this case,

$$
H_{n}=\frac{1}{2}\left(H_{1}^{2}+H_{2}^{2}\right)
$$

and straightforward computations can be done inside the abnormal triangle. Using the Poincaré coordinates $(q, H), H=$ $\left(H_{1}, H_{2}, H_{3}\right)$ and $H_{i}=\left\langle p, G_{i}(q)\right\rangle$. Indeed:

$$
\begin{aligned}
& \dot{H}_{1}=\mathrm{d} H_{1}\left(\vec{H}_{n}\right)=\left\{H_{1}, H_{2}\right\} H_{2}=H_{2} H_{3}, \\
& \dot{H}_{2}=\mathrm{d} H_{2}\left(\vec{H}_{n}\right)=\left\{H_{2}, H_{1}\right\} H_{1}=-H_{1} H_{3},
\end{aligned}
$$

Moreover

$$
\dot{H}_{3}=\mathrm{d} H_{3}\left(\vec{H}_{n}\right)=\left\{H_{3}, H_{1}\right\} H_{1}+\left\{H_{3}, H_{2}\right\} H_{2},
$$

where

$$
\begin{aligned}
& \left\{H_{3}, H_{1}\right\}(z)=\left\langle p,\left[\left[G_{1}, G_{2}\right], G_{1}\right](q)\right\rangle \\
& \left\{H_{3}, H_{2}\right\}(z)=\left\langle p,\left[\left[G_{1}, G_{2}\right], G_{2}\right](q)\right\rangle .
\end{aligned}
$$

At a contact point, $G_{1}, G_{2}, G_{3}$ form a frame, therefore we obtain

$$
\left[\left[G_{1}, G_{2}\right], G_{1}\right](q)=\sum_{i=1}^{3} \lambda_{i}(q) G_{i}(q)
$$

where $\lambda_{1}=\lambda_{2}=0, \frac{\partial f}{\partial \theta_{1}}=\lambda_{3} f$.

Similarly,

$$
\left[\left[G_{1}, G_{2}\right], G_{2}\right](q)=\sum_{i=1}^{3} \lambda_{i}^{\prime}(q) G_{i}(q)
$$

with

$$
\lambda_{1}^{\prime}=\lambda_{2}^{\prime}=0, \quad \frac{\partial f}{\partial \theta_{2}}=\lambda_{3}^{\prime} f
$$

We conclude that

$$
\begin{aligned}
& \dot{H}_{1}=H_{2} H_{3}, \quad \dot{H}_{2}=-H_{1} H_{3}, \\
& \dot{H}_{3}=H_{3}\left(\lambda_{3} H_{1}+\lambda_{3}^{\prime} H_{2}\right) .
\end{aligned}
$$

The associated one dimensional distribution can be analyzed by setting $\mathrm{d} s=H_{3} \mathrm{~d} t$ to obtain

$$
\frac{\mathrm{d} H_{1}}{\mathrm{~d} s}=H_{2}, \quad \frac{\mathrm{d} H_{2}}{\mathrm{~d} s}=-H_{1}, \quad \frac{\mathrm{d} H_{3}}{\mathrm{~d} s}=\lambda_{3} H_{1}+\lambda_{3}^{\prime} H_{2} .
$$

In particular, differentiating one more time the first relation of (7) with respect to $s$ and using the second relation, we have the harmonic oscillator $H_{1}^{\prime \prime}+H_{1}=0$.

Furthermore $H_{3}$ can be analyzed using the remaining equation (6). Observe that, with the approximation that $\lambda_{3}$ and $\lambda_{3}^{\prime}$ are constants, the equation takes the form

$$
\frac{\mathrm{d} H_{3}}{\mathrm{~d} s}=A \cos (s+\rho)
$$

for some constants $A$ and $\rho$. In those computations, we recognize the Heisenberg case, corresponding to $\lambda_{3}=\lambda_{3}^{\prime}=$ 0 .

Observe that when $q$ is not a contact point (that is $G_{2}=$ $\left[G_{1}, G_{2}\right] \in \operatorname{span}\left\{G_{1}, G_{2}\right\}$, in order to deal with the Martinet case, we can choose the frame $G_{1}^{\prime}, G_{2}^{\prime}$ and $G_{3}^{\prime}$, where $G_{1}^{\prime}=$ $G_{1}, G_{2}^{\prime}=G_{2}$ and $G_{3}^{\prime}=\frac{\partial}{\partial x}$.

Remark 3: Under the assumption of considering the simplified cost, we recover the policy described in Fig. 2 where the controls are given by trigonometric functions.

\section{NUMERICAL RESULTS}

The period $T$ is fixed to $2 \pi$ in our simulations. We use the HamPath software [9] for:

1) Solving the shooting equations associated with the problem and given by

$$
\begin{array}{ll}
x(0)=0, & x(2 \pi)=x_{f}, \\
\theta_{i}(0)=\theta_{i}(2 \pi), i=1,2 & p_{j}(0)=p_{j}(2 \pi), j=2,3 .
\end{array}
$$

2) Showing that the normal stroke is optimal. This is done by testing the nonexistence of conjugate points using the variational equation to compute the Jacobi fields. Recall that according to [7], given a reference curve $(q(t), p(t))$ solution of $\overrightarrow{H_{n}}$, a time $\left.\left.t_{c} \in\right] 0,2 \pi\right]$ is a conjugate time if there exists a Jacobi field $\delta z=(\delta q, \delta p)$, that is a non-zero solution of the variational equation

$$
\dot{\delta z}(t)=\frac{\partial \vec{H}_{n}}{\partial z}(q(t), p(t)) \delta z(t)
$$

such that $\delta q(0)=\delta q\left(t_{c}\right)=0$. We denote $\delta z_{i}=$ $\left(\delta q_{i}, \delta p_{i}\right), i=1, \ldots, n, n$-independent solutions of (8) with initial condition $\delta q_{i}(0)=0$. At time $t_{c}$ we have the following rank condition

$$
\operatorname{rank}\left\{\delta q_{1}\left(t_{c}\right), \ldots, \delta q_{n}\left(t_{c}\right)\right\}<n .
$$


1) Sample of various possible strokes: We present a sequence of numerical simulations, not taking into account the state constraints.

Fig.4-5-6 illustrate three different strokes illustrating the complexity of the model and are related to the generic classification of periodic planar curves [3].

Conjugate points are also computed to check the second order optimality conditions. There is no conjugate point on $[0,2 \pi]$ in the case of the simple loop whereas they appear for the limaçon case, the eight case and more complicated cases. Hence, the only candidates for optimality are the simple loops.
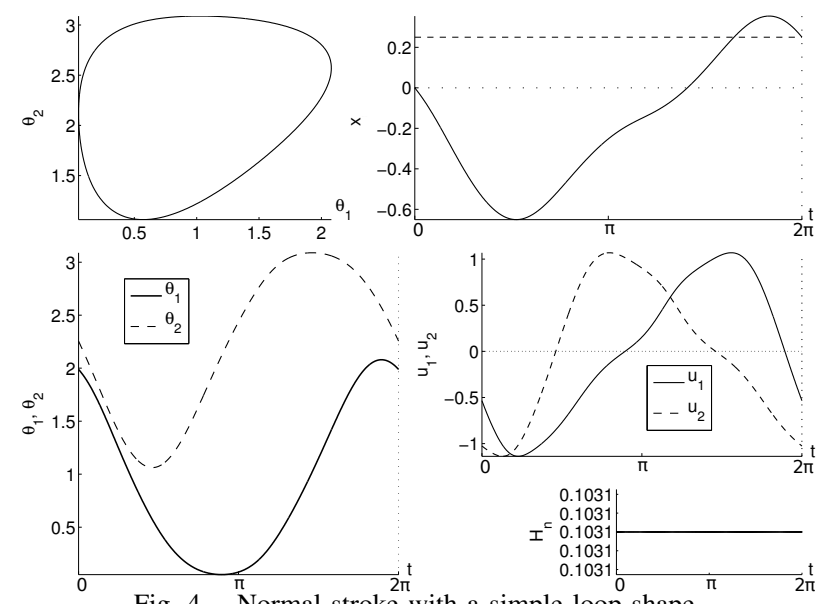

Fig. 4. $\quad \stackrel{\pi}{\text { Normal stroke }} \stackrel{2 \pi}{ }$ with a simple loop shape.
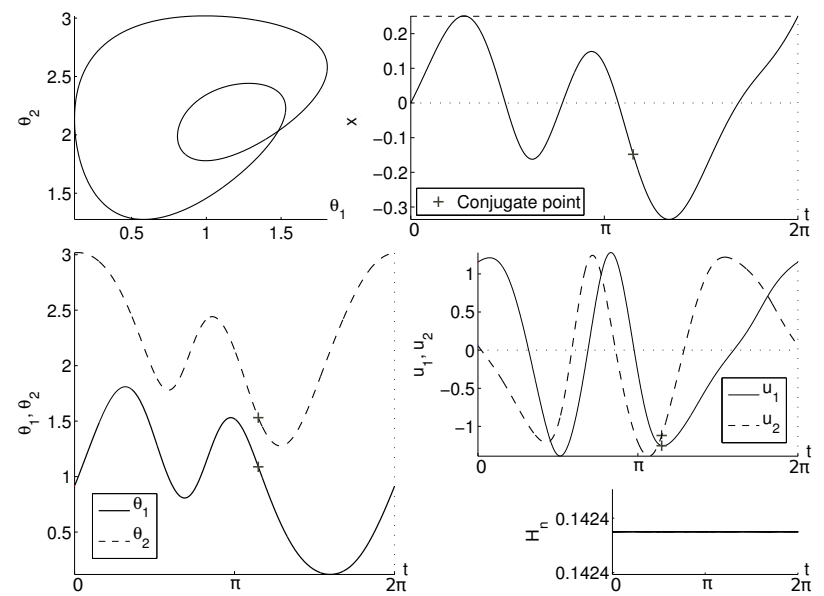

Fig. 5. Normal stroke with a limaçon with inner loop shape.

2) Optimal curves circumscribed in the triangle of constraints: We use a combination of the Bocop and HamPath softwares.

Bocop software: This software is suitable for taking into

account constraints on the state variables. Fig.7 gives numerical simulation with this software, describing a crawling normal stroke in accordance with the abnormal triangle policy. We use the Bocop software to initialize the simple shooting algorithm of the HamPath software.

HamPath software: This software cannot be directly applied to compute the optimal solution using the Maxi-

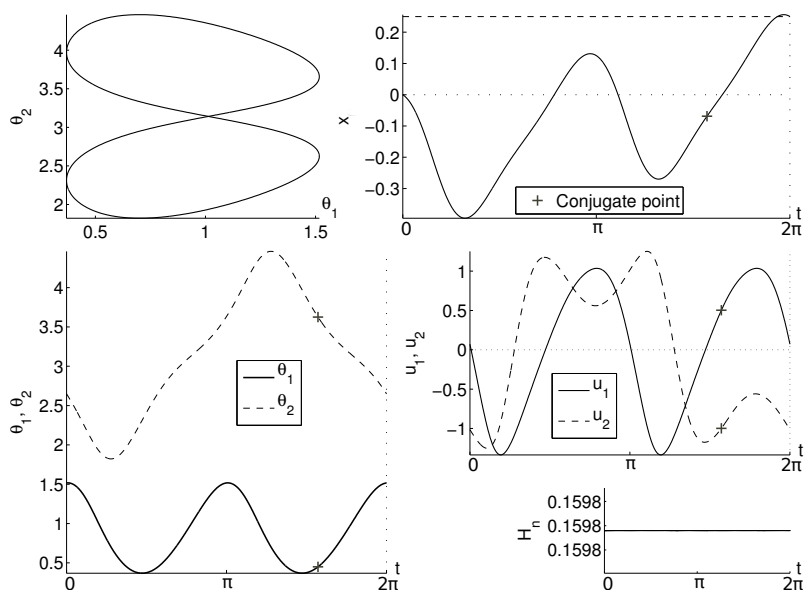

Fig. 6. Normal stroke with eight shape.

mum Principle with state constraints. Fig. 8 describes a normal stroke tangent to the boundary.

3) Geometric efficiency: To compare the different normal and abnormal solutions corresponding to different displacements and in relation with the SR-interpretation we represent the ratio $E=x / L$ where $L$ is the length of the stroke and $x$ is the corresponding displacement (this quantity does not depend upon the parameterization).

For the triangle, a displacement along the vertical or horizontal edge gives $x=\frac{2 \sqrt{3}}{3} \operatorname{arctanh}\left(\frac{\sqrt{3}}{3}\right)$ and along the hypotenuse $x=-\sqrt{2} \operatorname{arctanh}\left(\frac{\sqrt{2}}{2}\right)$ and the total displacement is $2.742 .10^{-1}$.

The length of a normal stroke $\gamma$ is $L(\gamma)=\int_{0}^{2 \pi} \sqrt{\langle\dot{q}, \dot{q}\rangle} \mathrm{d} t$ and easily computed using the energy level $H_{n}=\frac{1}{2}\langle\dot{q}, \dot{q}\rangle=$ $c$ and is $2 \pi \sqrt{2 c}$. The efficiency curve is presented in Fig.9. The normal strokes corresponding to the maximal efficiency is represented in Fig.9. For optimal strokes with constant mechanical power over time, the efficiency introduced in [17] is proportional to the square of the geometric efficiency $E$. Thus both efficiencies are maximized by the same strokes.

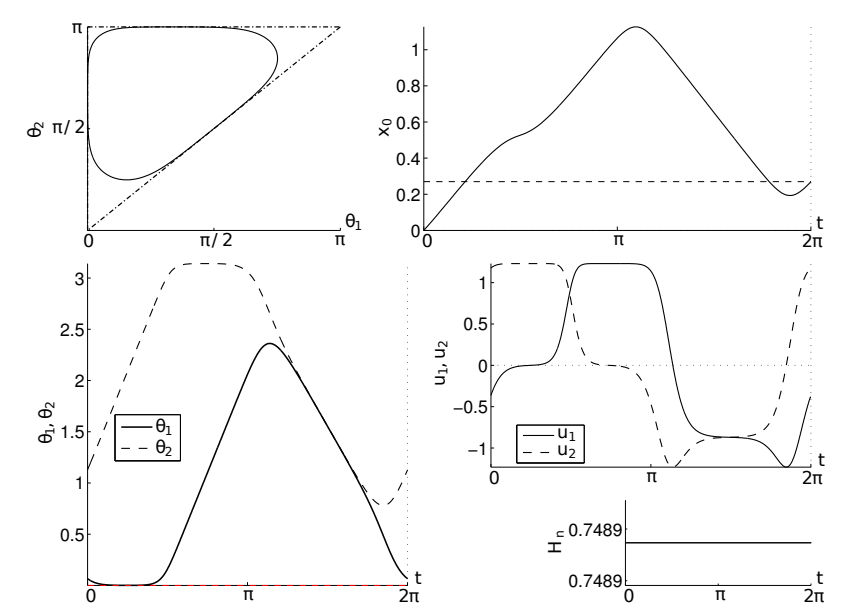

Fig. 7. Normal stroke with a simple loop shape where the constraints are satisfied, obtained with the Bocop software. 
Application. From our analysis we deduce that the (triangle) abnormal stroke is not optimal. Indeed, one can choose a normal stroke (inside the triangle) such that the displacement is $\bar{x} / 2$ with $\bar{x}=2.742$ and length $<\bar{L} / 2$ where $\bar{L}=$ length of the triangle. Applying twice the normal stroke, we obtain the same displacement $\bar{x}$ than with the abnormal stroke but with a length $<\bar{L}$.
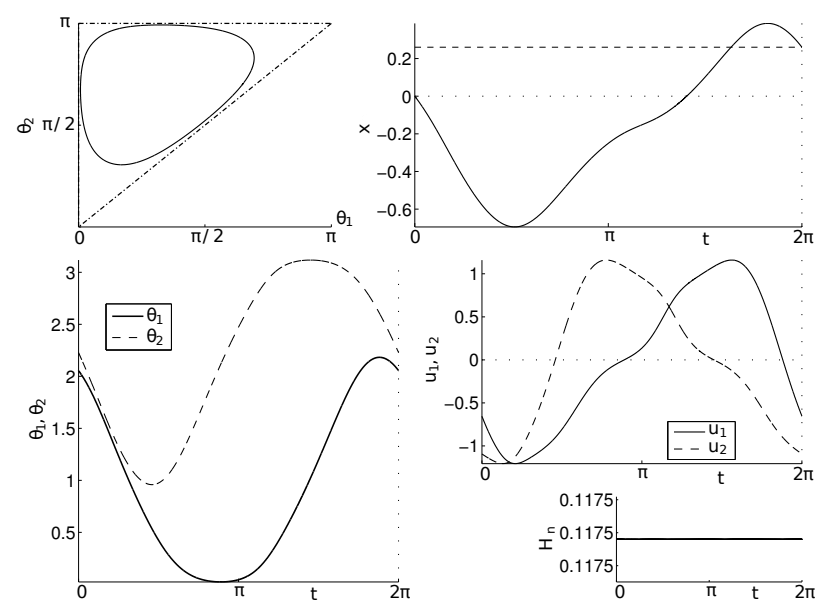

Fig. 8. Normal stroke with a simple loop shape where the constraints are satisfied.
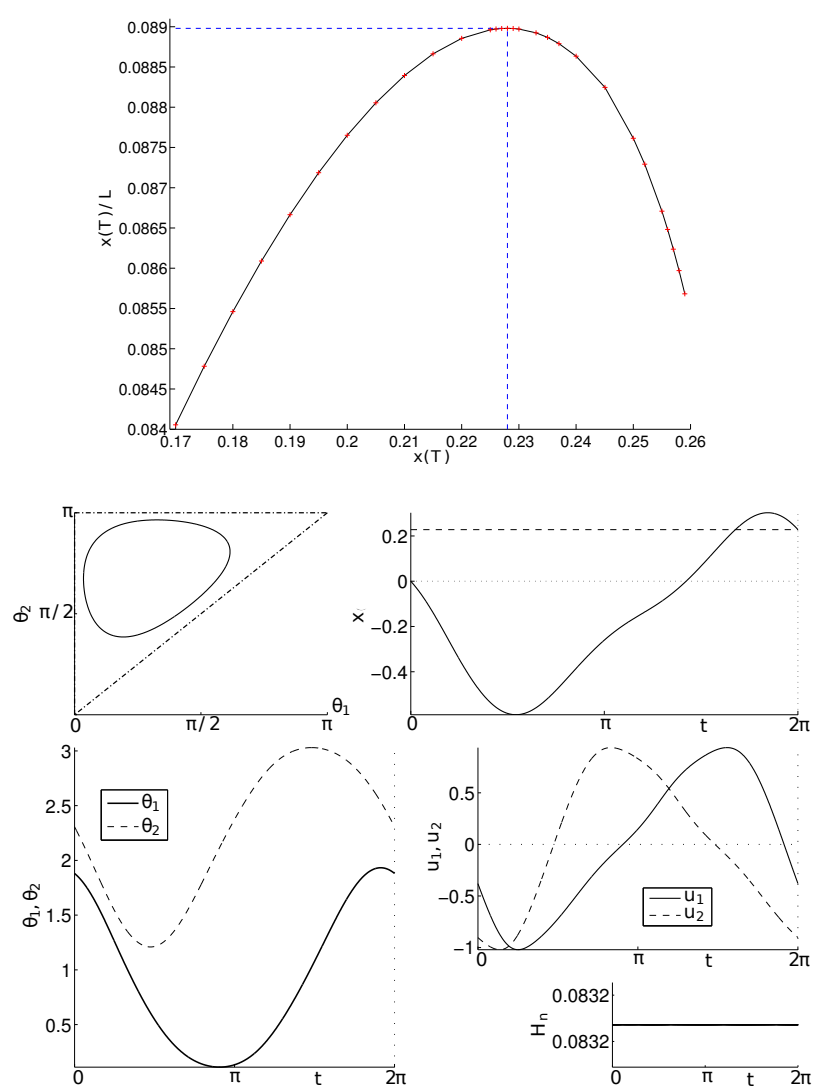

Fig. 9. Efficiency curve and the corresponding minimizing curve (bottom). The efficiency of the abnormal curve is $\mathbf{5 . 5 6} e^{-\mathbf{2}}$.

\section{CONCLUSION}

In this article we have investigated the copepod swimmer showing:

- from the micro-local point of view various topological strokes (simple loop, eight, limaçon, ...) are obtained confirming the complexity of the model.

- Using second order optimality conditions only simple loops are candidates for optimality.

- The abnormal triangle forming the boundary of the domain is shown to be not optimal, using the computation of the efficiency.

This numerical study opens the road to analyze the more complicated Purcell three-link swimmer, see [5] for preliminary results.

\section{REFERENCES}

[1] Alouges, F., DeSimone, A., Giraldi, L., Zoppello, M.: Self-propulsion of slender microswimmers by curvature control: N-link swimmers. Int. J. Nonlinear Mech. 56 (2013)

[2] Alouges, F., DeSimone, A., Lefebvre, A.: Optimal strokes for low Reynolds number swimmers: an example. J. Nonlinear Sci. 18, 277$302(2008)$

[3] Berger, M.: La taxonomie des courbes. Pour la science, 297 56-63 (2002)

[4] Becker, L.E., Koehler, S.A., Stone, H.A.: On self-propulsion of micromachines at low Reynolds number: Purcell's three-link swimmer. J. Fluid Mech. 490, 15-35 (2003)

[5] Bettiol, P., Bonnard, B., Giraldi, L., Martinon, P., Rouot, J.: The Purcell Three-link swimmer: some geometric and numerical aspects related to periodic optimal controls. Rad. Ser. Comp. App. 18, Variational Methods, Ed. by M. Bergounioux et al. (2016)

[6] Bonnans, F., Giorgi, D., Maindrault, S., Martinon, P., Grélard, V.: Bocop - A collection of examples, Inria Research Report, ProjectTeam Commands. 8053 (2014)

[7] Bonnard, B., Chyba, M.: Singular trajectories and their role in control theory. Mathématiques \& Applications 40, Springer-Verlag, Berlin (2003)

[8] Bonnard, B., Faubourg, L., Trélat, E.: Mécanique céleste et contrôle des véhicules spatiaux. Mathématiques \& Applications 51, SpringerVerlag Berlin (2006)

[9] Cots, O.: Contrôle optimal géométrique: méthodes homotopiques et applications. PhD thesis, Université de Bourgogne (2012)

[10] Giraldi J., Martinon, P., Zoppello, M.: Optimal design of the three-link Purcell swimmer. Phys. Rev. E 91 (2015)

[11] Gray, J., Hancock, G. J.: The propulsion of sea-urchin spermatozoa. J. Exp. Biol. 32, 802-814 (1955)

[12] Hakavuori, E., Le Donne, E.: Non-minimality of corners in subRiemannian geometry. Preprint (2015)

[13] Lauga, E., Powers, T.R.: The hydrodynamics of swimming microorganisms. Rep. Progr. Phys. 72, 9 (2009)

[14] Passov, E., Or, Y.: Supplementary notes to: Dynamics of Purcell's three-link microswimmer with a passive elastic tail. EPJ E 35, 1-9 (2012)

[15] Lenz, P. H., Takagi, D., Hartline,D. K.: Choreographed swimming of copepod nauplii. J. R. Soc. Interface 12, 20150776 (2015)

[16] Purcell, E.M.: Life at low Reynolds number. Am. J. Phys. 45, 3-11 (1977)

[17] Takagi, D.: Swimming with stiff legs at low Reynolds number. Phys. Rev. E 92. (2015)

[18] Tam, D., Hosoi, A.E.: Optimal Stroke Patterns for Purcell Three-Link Swimmer. Phys. Rev. Lett. 98 (2007)

[19] Taylor, G.I.: Analysis of the swimming of microscopic organisms. Proc. Roy. Soc. London. Ser. A. 209, 447-461 (1951)

[20] Zhitomirskiı̌, M.: Typical singularities of differential 1-forms and Pfaffian equations. American Mathematical Society, Providence, RI. 113 (1992) 